\title{
An Analytical Solution for Effect of Magnetic Field and Initial Stress on an Infinite Generalized Thermoelastic Rotating Nonhomogeneous Diffusion Medium
}

\author{
S. R. Mahmoud ${ }^{1,2}$ \\ ${ }^{1}$ Mathematics Department, Science Faculty, King Abdulaziz University, Jeddah 21589, Saudi Arabia \\ ${ }^{2}$ Mathematics Department, Science Faculty, Sohag University, Sohag 82524, Egypt
}

Correspondence should be addressed to S. R. Mahmoud; samsam73@yahoo.com

Received 12 July 2013; Revised 11 October 2013; Accepted 19 October 2013

Academic Editor: Hasan Ali Yurtsever

Copyright (C) 2013 S. R. Mahmoud. This is an open access article distributed under the Creative Commons Attribution License, which permits unrestricted use, distribution, and reproduction in any medium, provided the original work is properly cited.

\begin{abstract}
The problem of generalized magneto-thermoelastic diffusion in an infinite rotating nonhomogeneity medium subjected to certain boundary conditions is studied. The chemical potential is also assumed to be a known function of time at the boundary of the cavity. The analytical expressions for the displacements, stresses, temperature, concentration, and chemical potential are obtained. Comparison was made between the results obtained in the presence and absence of diffusion. The results indicate that the effect of nonhomogeneity, rotation, magnetic field, relaxation time, and diffusion is very pronounced.
\end{abstract}

\section{Introduction}

Diffusion can be defined as the spontaneous migration of substances from regions of high concentration to regions of low concentration. There is now a great deal of interest in the study of this phenomenon due to its many applications in geophysics and industrial applications. Thermodiffusion in the solids is one of the transport processes which has great practical importance. Thermodiffusion in an elastic solid is due to the coupling of the fields of temperature, mass diffusion, and that of strain. This mater has attracted the attention of many researchers such as [1-5]. Wave propagation in rotating and nonhomogeneous media was studied by Abd-Alla et al. [6-8]. The extended thermoelasticity theory, introducing one relaxation time in the thermoelastic process, was proposed by Lord and Shulman [9]. In this theory, a modified law of heat conduction including both the heat flux and its time derivative replaces conventional Fourier's law. The heat equation associated with this is a hyperbolic one and hence automatically eliminates the paradox of infinite speeds of propagation inherent in the coupled theory of thermoelasticity. This theory was extended by Dhaliwal and Sherief [10] to include the anisotropic case. Abd-Alla and
Mahmoud [11] investigated the magneto-thermoelastic problem in rotating nonhomogeneous orthotropic hollow cylinder under the hyperbolic heat conduction model. Mahmoud [12] investigated wave propagation in cylindrical poroelastic dry bones.

Kumar and Devi [13] studied deformation in porous thermoelastic material with temperature dependent properties. Othman et al. [14] presented the study of the two-dimensional problems of generalized thermoelasticity with one relaxation time with the modulus of elasticity being dependent on the reference temperature for nonrotating and rotating medium, respectively. Kumar and Gupta [15] investigated deformation due to inclined load in an orthotropic micropolar thermoelastic medium with two relaxation times. The temperaturerate dependent theory of thermoelasticity, which takes into account two relaxation times, was developed by Green and Lindsay [16]. Abd-Alla et al. [17, 18] investigated radial vibrations in a nonhomogeneous orthotropic elastic medium subjected to rotation and gravity field. Sherief et al. [19] developed the generalized theory of thermoelastic diffusion with one relaxation time, which allows the finite speed of propagation waves. Sherief and Saleh [20] investigated the problem of a thermoelastic half-space in the context 
of the theory of generalized thermoelastic diffusion with one relaxation time. The reflection of SV waves from the free surface of an elastic solid in generalized thermoelastic diffusion was discussed by Singh [21]. Kumar and Kansal [22] discussed the propagation of Lamb waves in transversely isotropic thermoelastic diffusive plates. Thermomechanical response of generalized thermoelastic diffusion with one relaxation time due to time harmonic sources was discussed by Ram et al. [23]. Aouadi [24] examined the thermoelastic diffusion problem for an infinite elastic body with spherical cavity. Abd-Alla and Mahmoud [25] investigated analytical solution of wave propagation in nonhomogeneous orthotropic rotating elastic media. Othman et al. [26] discussed the effect of diffusion in a two-dimensional problem of generalized thermoelasticity with Green-Naghdi theory. Xia et al. [27] studied the influence of diffusion on generalized thermoelastic problems of infinite body with a cylindrical cavity. Deswal and Kalkal [28] studied the two-dimensional generalized electromagneto-thermoviscoelastic problem for a half-space with diffusion. Abd-Alla and Abo-Dahab [29] found the time-harmonic sources in a generalized magnetothermo-viscoelastic continuum with and without energy dissipation. Mahmoud [30] discussed influence of rotation and generalized magnetothermoelastic on Rayleigh waves in a granular medium under effect of initial stress and gravity field. Abd-Alla et al. [31, 32] studied the generalized magnetothermoelastic Rayleigh waves in a granular medium under the influence of a gravity field and initial stress.

In the present investigation, the temperature, displacements, stresses, diffusion, and concentration as well as chemical potential are obtained in the physical domain using the harmonic vibrations. Also, study of the interaction between the processes of elasticity, nonhomogeneity, rotation, magnetic field, initial stress, heat, and diffusion in an infinite elastic solid with a spherical cavity in the context of the theory of generalized thermoelastic diffusion is presented.

\section{Formulation of the Problem}

Consider a perfect electric conductor and linearized Maxwell equations governing the electromagnetic field in the absence of the displacement current (SI) in the form as in Kraus [33]. Applying an initial magnetic field vector $\vec{H}\left(0,0, H_{0}\right)$ in spherical coordinates $(r, \theta, \phi), \vec{u}=(u(r, t), 0,0)$. One will consider a nonhomogeneous, isotropic medium, occupying the region $a \leq r \leq b$, where a is the radius of the spherical cavity. The strain tensor has the following components:

$$
\begin{gathered}
e_{i j}=\frac{1}{2}\left(u_{i, j}+u_{j, i}\right), \\
\omega_{i j}=\frac{1}{2}\left(u_{j, i}-u_{i, j}\right), \\
e_{k k}=\frac{1}{r^{2}} \frac{\partial}{\partial r}\left(r^{2} u\right), \quad i, j, k=1,2,3, \\
e_{r r}=\frac{\partial u}{\partial r}, \quad e_{\theta \theta}=e_{\phi \phi}=\frac{u}{r} .
\end{gathered}
$$

The cubical dilatation is given by $\left(1 / r^{2}\right)(\partial / \partial r)\left(r^{2} u\right)$, where the nonvanishing displacement component is the radial one $u_{r}=u(r, t)$. The elastic medium is rotating uniformly with an angular velocity $\vec{\Omega}=\Omega \vec{n}$, where $\vec{n}$ is a unit vector representing the direction of the axis of rotation. The displacement equation of motion in the rotating frame has two additional terms: $\vec{\Omega} \times(\vec{\Omega} \times \vec{u})$ which is the centripetal acceleration due to time varying motion only, and $2 \vec{\Omega} \times \overrightarrow{\dot{u}}$ is the Coriolis acceleration, where $\vec{\Omega}=(0, \Omega, 0)$. Following Sherief's theory of generalized thermoelastic diffusion [19] and Sherief and Saleh [20], one is going to study an isotropic nonhomogeneous elastic medium which suffers thermal shock. Due to spherical symmetry, the stress-displacementtemperature-diffusion relation or constitutive equations are given by

$$
\begin{aligned}
\sigma_{r r}= & \left(2 \mu+P_{1}\right) \frac{\partial u}{\partial r}+\left(\lambda+P_{1}\right) \frac{1}{r^{2}} \frac{\partial}{\partial r}\left(r^{2} u\right) \\
& -\beta_{1}\left(\theta-\theta_{0}\right)-\beta_{2} C, \\
\sigma_{\phi \phi}=\sigma_{\theta \theta}= & \left(2 \mu+P_{1}\right) \frac{u}{r}+\left(\lambda+P_{1}\right) \frac{1}{r^{2}} \frac{\partial}{\partial r}\left(r^{2} u\right) \\
& -\beta_{1}\left(\theta-\theta_{0}\right)-\beta_{2} C .
\end{aligned}
$$

The chemical-displacement-temperature-diffusion relation is given by

$$
P=-\beta_{2} \frac{1}{r^{2}} \frac{\partial}{\partial r}\left(r^{2} u\right)+b C-c\left(\theta-\theta_{0}\right) .
$$

The governing equation for an isotropic nonhomogeneous elastic solid with generalized magneto-thermoelastic diffusion under effect of rotation is given by

$$
\begin{gathered}
\frac{\partial\left(1 / r^{2}\right)(\partial / \partial r)\left(r^{2} u\right)}{\partial r}-\frac{\beta_{2}}{h_{0}} \frac{\partial C}{\partial r}-\frac{\beta_{1}}{h_{0}} \frac{\partial\left(\theta-\theta_{0}\right)}{\partial r}+F_{r} \\
=\frac{\rho}{h_{0}}\left[\frac{\partial^{2} u}{\partial t^{2}}-\Omega^{2} u-2 \Omega \frac{\partial u}{\partial t}\right],
\end{gathered}
$$

where $\lambda$ and $\mu$ are Lame's elastic constants, $\delta_{i j}$ is Kronecker's delta, $P_{1}$ is the initial stress, $\rho$ is the density of the medium, and $\vec{F}$ is defined as Lorentz's force which may be written as

$$
\vec{F}=\mu_{e}(\vec{J} \times \vec{H})=\left(\mu_{e} H_{0}^{2} \frac{\partial}{\partial r}\left(\frac{\partial u}{\partial r}+\frac{2 u}{r}\right), 0,0\right),
$$

where $\mu_{e}$ is the magnetic permeability, $\vec{H}$ is the magnetic field vector, $\vec{J}$ is the electric current density, $\vec{u}$ is the displacement vector, and $t$ is the time.

Equation of heat conduction is given by

$$
K \nabla^{2} \theta=\left(\frac{\partial}{\partial t}+\tau \frac{\partial^{2}}{\partial t^{2}}\right)\left(\rho c_{v} \theta+\theta_{0} \beta_{1} \frac{1}{r^{2}} \frac{\partial}{\partial r}\left(r^{2} u\right)+c \theta_{0} C\right)
$$

where the Laplacian operator $\nabla^{2}$ is given by $\nabla^{2}=\left(\partial^{2} / \partial r^{2}\right)+$ $(2 / r)(\partial / \partial r)$ and $h_{0}=2 \mu+\lambda+\left(P_{1} / 2\right)+\mu_{e} H_{0}^{2}, h_{0}$ is the 
coefficient of linear diffusion expansion, $K$ is the thermal conductivity, $\theta$ is the absolute temperature, $\theta_{0}$ is the initial uniform temperature, and $\left|\left(\theta-\theta_{0}\right) / \theta_{0}\right| \ll 1$.

Equation of conservation of mass diffusion may be written as

$$
D \beta_{2} \nabla^{2} \frac{1}{r^{2}} \frac{\partial}{\partial r}\left(r^{2} u\right)+D c \nabla^{2} \theta+\left(\frac{\partial}{\partial t}+\tau \frac{\partial^{2}}{\partial t^{2}}\right) C=D b \nabla^{2} C
$$

where $\tau$ is the diffusion relaxation time, $\tau_{0}$ is the thermal relaxation time, $\alpha_{t}$ is the coefficient of linear thermal expansion, and $\theta_{0}$ is constant, where $\beta_{1}=(3 \lambda+2 \mu) \alpha_{t}, \beta_{2}=$ $(3 \lambda+2 \mu) \alpha_{c}, \quad \sigma_{i j}$ are the components of the stress tensor, $\tau_{i j}$ are the components of stress tensor, $b$ and $c$ are the measures of thermodiffusion and diffusive effects, $C$ is the concentration, $C_{v}$ is the specific heat at constant strain, $D$ is the diffusive coefficient, and $e_{i j}$ are the components of the strain tensor. The thermal relaxation time $\tau_{0}$ will ensure that the heat conduction equation will predict finite speed of heat propagation. The diffusion relaxation time $\tau$, which will ensure the equation satisfied by the concentration $C$, will also predict finite speed of propagation of matter from one medium to the other.

\section{Dimensionless Quantities}

Introduce the following nondimensional parameters:

$$
\begin{gathered}
r^{*}=c_{1} \eta_{0} r, \quad u^{*}=c_{1} \eta_{0} u, \quad T=\frac{\beta_{1}\left(\theta-\theta_{0}\right)}{h_{0}}, \\
C^{*}=\frac{\beta_{2} C}{h_{0}}, \quad \Omega^{*}=\frac{\Omega}{c_{1}^{2} \eta_{0}}, \quad \sigma_{i j}^{*}=\frac{\sigma_{i j}}{h_{0}}, \\
P^{*}=\frac{P}{\beta_{2}}, \quad t^{*}=c_{1}^{2} \eta_{0} t, \quad \tau_{0}^{*}=c_{1}^{2} \eta_{0} \tau_{0}, \\
\tau^{*}=c_{1}^{2} \eta_{0} \tau, \quad \eta_{0}=\frac{\rho c_{v}}{K}, \quad c_{1}^{2}=\frac{h_{0}}{\rho} .
\end{gathered}
$$

The elastic constants $\lambda, \mu$ and the density $\rho$ of nonhomogeneous material in form [32] are as follows:

$$
\begin{gathered}
\lambda=r^{2 m} \lambda_{0}, \quad \mu=r^{2 m} \mu_{0}, \quad \mu_{h}=r^{2 m} \mu_{0}, \\
\rho=r^{2 m} \rho_{0}, \quad p^{*}=p_{0}^{*} r^{2 m} .
\end{gathered}
$$

Using the above non-dimensional parameters and (9) in (10)-(14), the non-dimensional system becomes

$$
\begin{gathered}
\frac{\partial\left(1 / r^{2}\right)(\partial / \partial r)\left(r^{2} u\right)}{\partial r}-\frac{\partial C}{\partial r}-\frac{\partial T}{\partial r}=\frac{\partial^{2} u}{\partial t^{2}}-\Omega^{2} u-2 \Omega \frac{\partial u}{\partial t} \\
\nabla^{2} T=\left(\frac{\partial}{\partial t}+\tau_{0} \frac{\partial^{2}}{\partial t^{2}}\right)\left(T+\varepsilon_{1} \frac{1}{r^{2}} \frac{\partial}{\partial r}\left(r^{2} u\right)+\varepsilon_{2} C\right), \\
\nabla^{2} \frac{1}{r^{2}} \frac{\partial}{\partial r}\left(r^{2} u\right)+h_{4} \nabla^{2} T+h_{6}\left(\frac{\partial}{\partial t}+\tau \frac{\partial^{2}}{\partial t^{2}}\right) C=h_{5} \nabla^{2} C
\end{gathered}
$$

$$
\begin{gathered}
\sigma_{r r}=h_{1} \frac{\partial u}{\partial r}+h_{2} \frac{1}{r^{2}} \frac{\partial}{\partial r}\left(r^{2} u\right)-T-C, \\
\sigma_{\theta \theta}=h_{1} \frac{u}{r}+h_{2} \frac{1}{r^{2}} \frac{\partial}{\partial r}\left(r^{2} u\right)-T-C, \\
\sigma_{\phi \phi}=h_{1} \frac{u}{r}+h_{2} \frac{1}{r^{2}} \frac{\partial}{\partial r}\left(r^{2} u\right)-T-C, \\
P=-\frac{1}{r^{2}} \frac{\partial}{\partial r}\left(r^{2} u\right)+h_{3} C-h_{4} T
\end{gathered}
$$

where

$$
\begin{gathered}
\varepsilon_{1}=\frac{\beta_{1}^{2} T_{0} m}{h_{0} \rho c_{v}}, \quad \varepsilon_{2}=\frac{\beta_{1} c T_{0} h_{0}(m+2)}{\beta_{2}}, \quad h_{1}=\frac{2 \mu}{h_{0}}, \\
h_{2}=\frac{\lambda}{h_{0}}, \quad h_{3}=\frac{m b h_{0}}{\beta_{2}^{2}}, \quad h_{4}=\frac{c h_{0}}{\beta_{1} \beta_{2}}, \\
h_{5}=\frac{D b h_{0}}{\beta_{2}}, \quad h_{6}=\frac{2 m+h_{0}}{\beta_{2}^{2} D \eta_{0}} .
\end{gathered}
$$

\section{Boundary Conditions}

The nonhomogeneous initial conditions are supplemented by the following boundary conditions. The cavity surface is traction free:

$$
\sigma_{r r}(r, t)+\bar{\tau}_{r r}(r, t)=0, \quad r=a .
$$

The cavity surface is subjected to a thermal shock

$$
T(a, t)=T_{0} H(t)
$$

where $H(t)$ is the Heaviside unit step function. The chemical potential is also assumed to be a known function of time at the cavity surface:

$$
P(a, t)=P_{0} H(t), \quad P_{0} \text { is real constant. }
$$

The displacement function is as follows:

$$
u(r, t)=0, \quad r=a .
$$

\section{Solution of the Problem}

In this section, one obtains the analytical solution of the problem for a spherical region with boundary conditions by taking the harmonic vibrations. One assumes that the solution of (10)-(12) as follows:

$$
\begin{array}{ll}
C(r, t)=C^{\prime}(r) e^{i \omega t}, & T(r, t)=T^{\prime}(r) e^{i \omega t}, \\
u(r, t)=u^{\prime}(r) e^{i \omega t} . & e(r, t)=E^{\prime}(r) e^{i \omega t},
\end{array}
$$




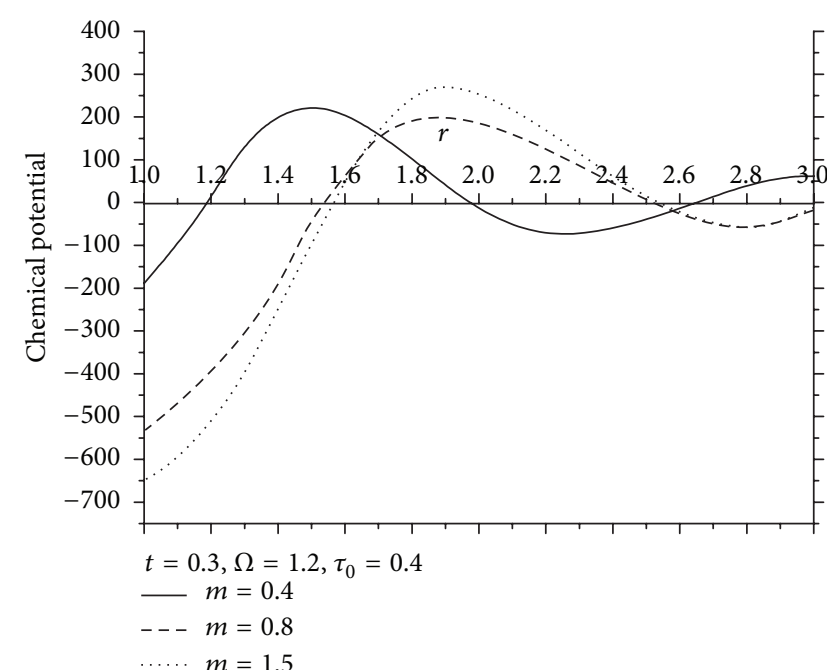

(a)

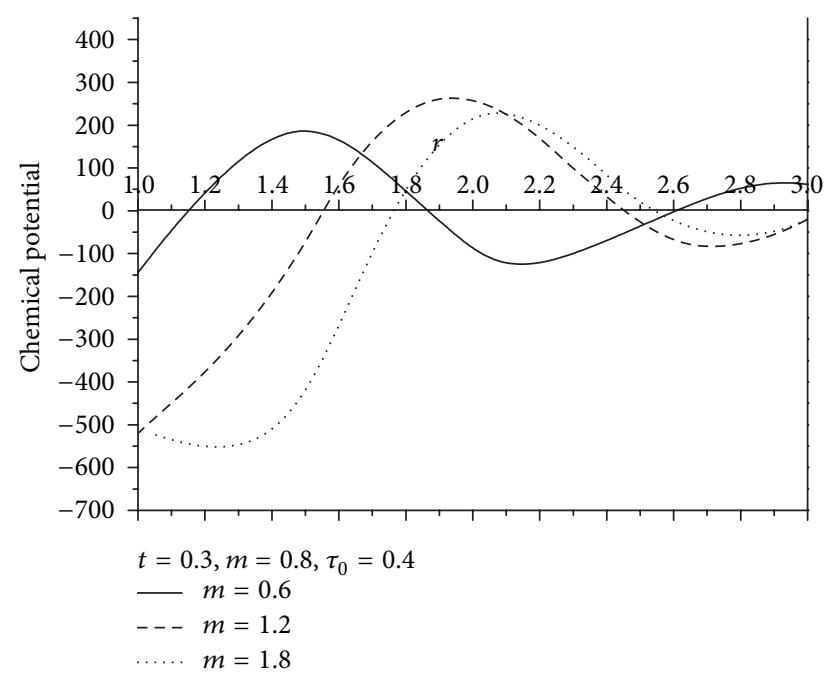

(b)

FIgURE 1: Variation of chemical potential $P$ with radius $r$ (thermoelastic diffusion nonhomogeneity medium).

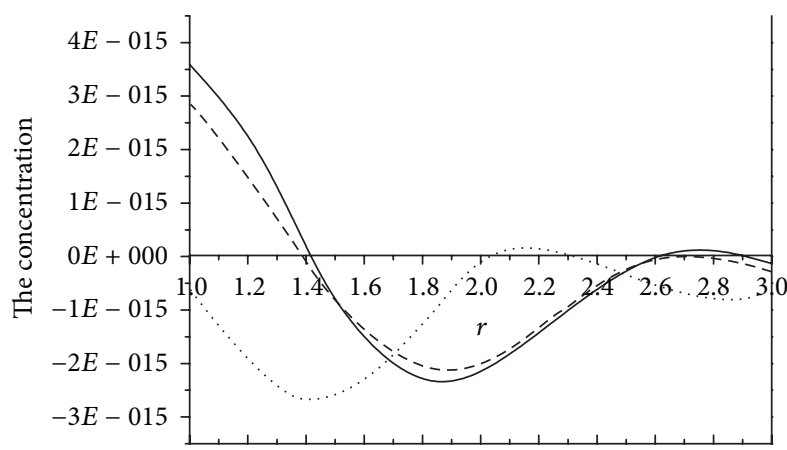

$t=0.3, \Omega=1.2, \tau_{0}=0.4$

$-m=0.4$

- - $m=0.8$

… $m=1.5$

(a)

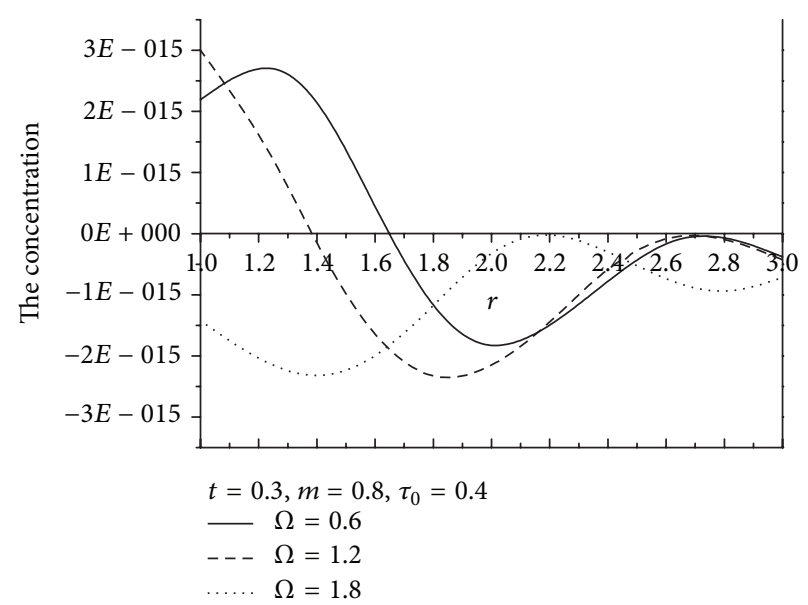

(b)

FIGURE 2: Variation of concentration $C$ with radius $r$ (thermoelastic diffusion nonhomogeneity medium).

where $e=(\partial u / \partial r)+(2 u / r)=\left(1 / r^{2}\right)(\partial / \partial r)\left(r^{2} u\right)$.

Substituting (17a) and (17b) into (10)-(12) yields

$$
\begin{gathered}
\frac{\partial E^{\prime}}{\partial r}-\frac{\partial C^{\prime}}{\partial r}-\frac{\partial T^{\prime}}{\partial r}=\beta u^{\prime}, \\
\nabla^{2} T^{\prime}=k_{1}\left(T^{\prime}+\varepsilon_{1} E^{\prime}+\varepsilon_{2} C^{\prime}\right), \\
\nabla^{2} E^{\prime}+h_{4} \nabla^{2} T^{\prime}+h_{6} k_{2} G=h_{5} \nabla^{2} C^{\prime} .
\end{gathered}
$$

Applying the operator Laplacian operator $\nabla^{2}$ to (18), we obtain

$$
\left(\nabla^{2}-\beta\right) E^{\prime}=\nabla^{2} C^{\prime}+\nabla^{2} T^{\prime}
$$

From (19)-(21), we obtain

$$
\left(\nabla^{6}+b_{1} \nabla^{4}+b_{2} \nabla^{2}+b_{3}\right)\left(E^{\prime}, T^{\prime}, C^{\prime}\right)=0
$$

where

$$
\begin{aligned}
b_{1}= & \frac{-1}{\left(h_{5}-1\right)} \\
& \times\left[k_{2} h_{6}+k_{1}\left(h_{5}+\varepsilon_{2} h_{4}\right)+\beta h_{5}+k_{1}\left(\varepsilon_{1} h_{5}+\varepsilon_{2}\right)\right], \\
b_{2}= & \frac{1}{\left(h_{5}-1\right)} \\
& \times\left[k_{1} k_{2} h_{6}+\beta\left(k_{2} h_{6}+k_{1}\left(h_{5}+\varepsilon_{2} h_{4}\right)\right)+k_{1} k_{2} \varepsilon_{1} h_{6}\right], \\
& b_{3}=\frac{-\beta k_{1} k_{2} h_{6}}{\left(h_{5}-1\right)}, \quad k_{1}=i \omega\left(1+i \omega \tau_{0}\right), \\
k_{2}= & i \omega(1+i \omega \tau), \quad \beta=-\left(\omega^{2}+\Omega^{2}+2 i \omega \Omega\right) .
\end{aligned}
$$




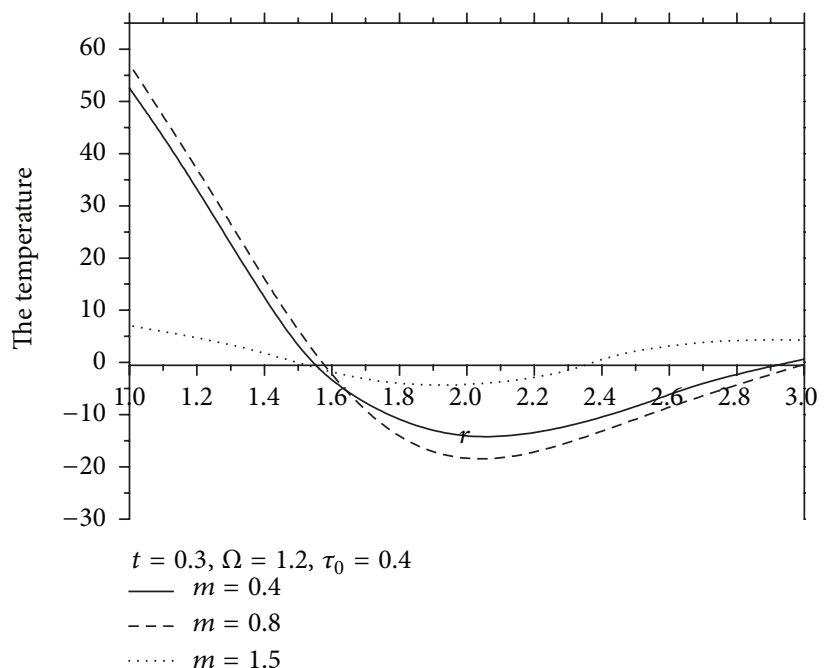

(a)

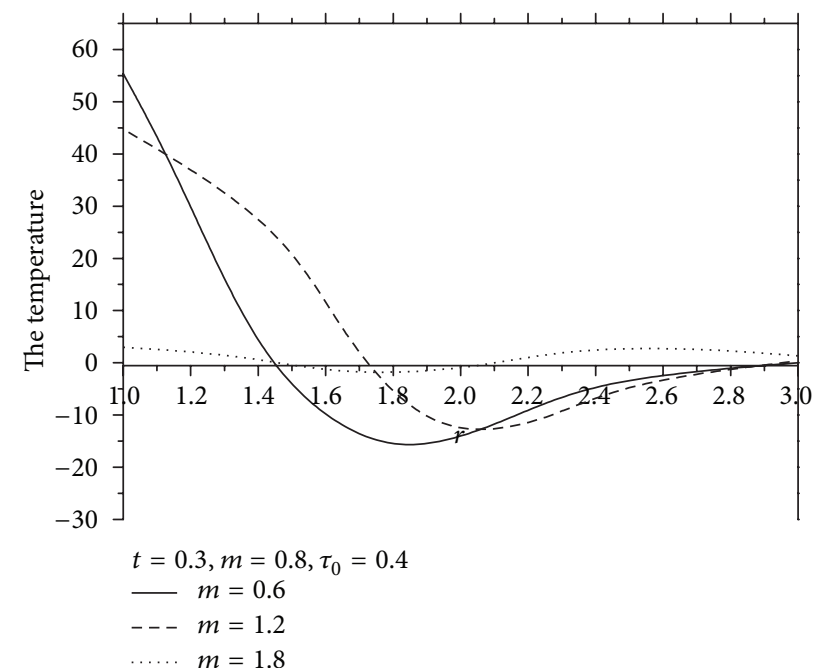

(b)

FIgURE 3: Variation of temperature $\theta$ with radius $r$ (thermoelastic diffusion nonhomogeneity medium).

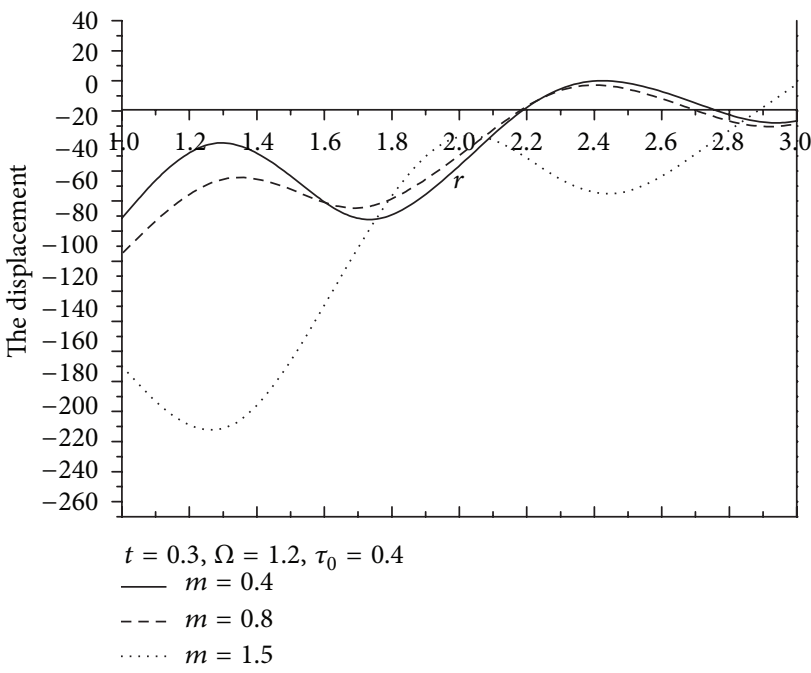

(a)

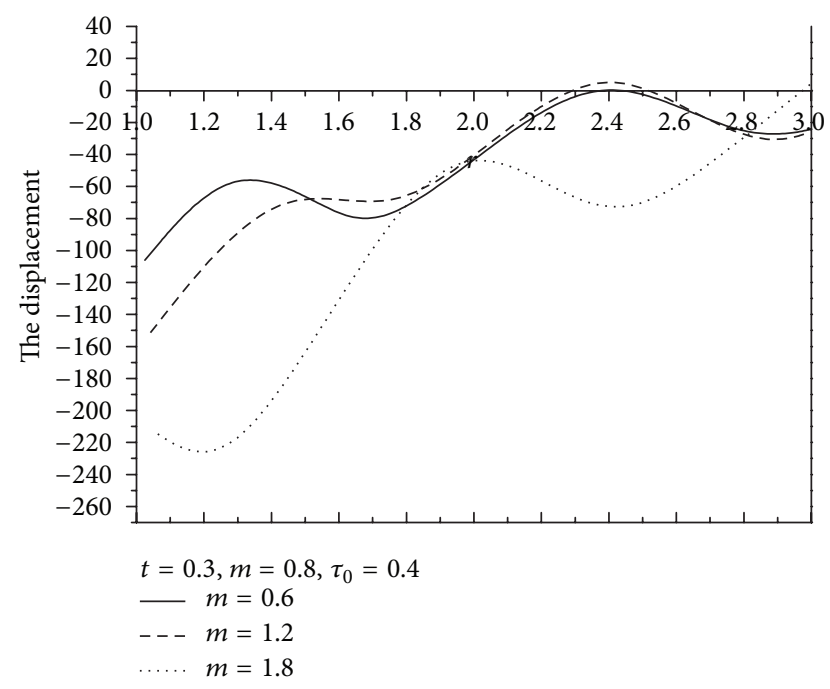

(b)

FIgURE 4: Variation of displacement $u$ with radius $r$ (thermoelastic diffusion nonhomogeneity medium).

Equation (22) can be factorized as

$$
\left(\nabla^{2}+Q_{1}^{2}\right)\left(\nabla^{2}+Q_{2}^{2}\right)\left(\nabla^{2}+Q_{3}^{2}\right)\left(E^{\prime}, T^{\prime}, C^{\prime}\right)=0,
$$

where $Q_{1}^{2}, Q_{2}^{2}$, and $Q_{3}^{2}$ are the roots of the characteristic equation

$$
Q^{6}+b_{1} Q^{4}+b_{2} Q^{2}+b_{3}=0 .
$$

The solution of (24) which is bounded at infinity is given by

$$
\begin{aligned}
& T^{\prime}(r, \omega)=\frac{1}{\sqrt{r}} \sum_{j=1}^{3} B_{j}(\omega) K_{1 / 2}\left(Q_{j} r\right), \\
& E^{\prime}(r, \omega)=\frac{1}{\sqrt{r}} \sum_{j=1}^{3} B_{j}^{\prime}(\omega) K_{1 / 2}\left(Q_{j} r\right), \\
& C^{\prime}(r, \omega)=\frac{1}{\sqrt{r}} \sum_{j=1}^{3} B_{j}^{\prime \prime}(\omega) K_{1 / 2}\left(Q_{j} r\right),
\end{aligned}
$$

where $B_{j}, B_{j}^{\prime}$, and $B_{j}^{\prime \prime}$ are parameters depending only on $\omega$ and $K_{1 / 2}$ is the modified spherical Bessel function of the second 


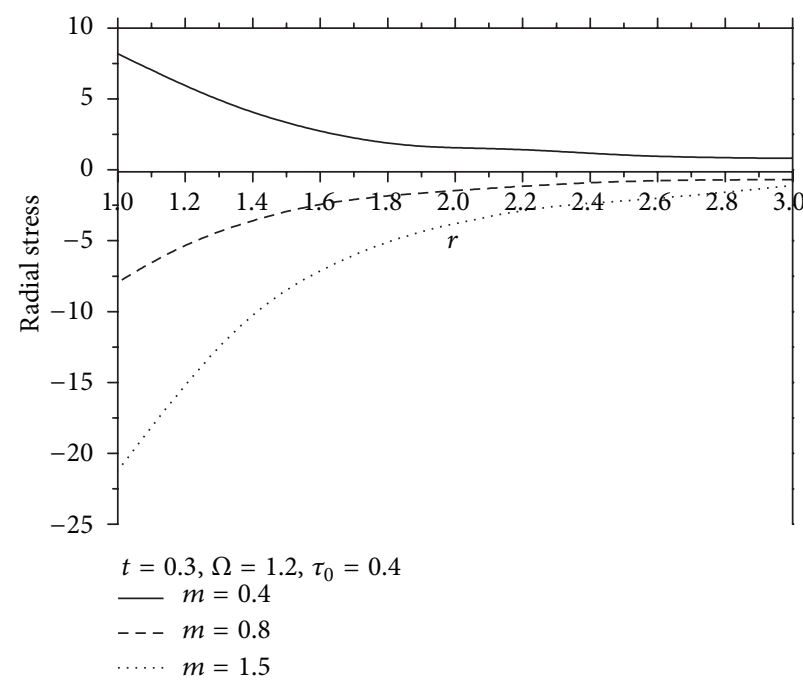

(a)

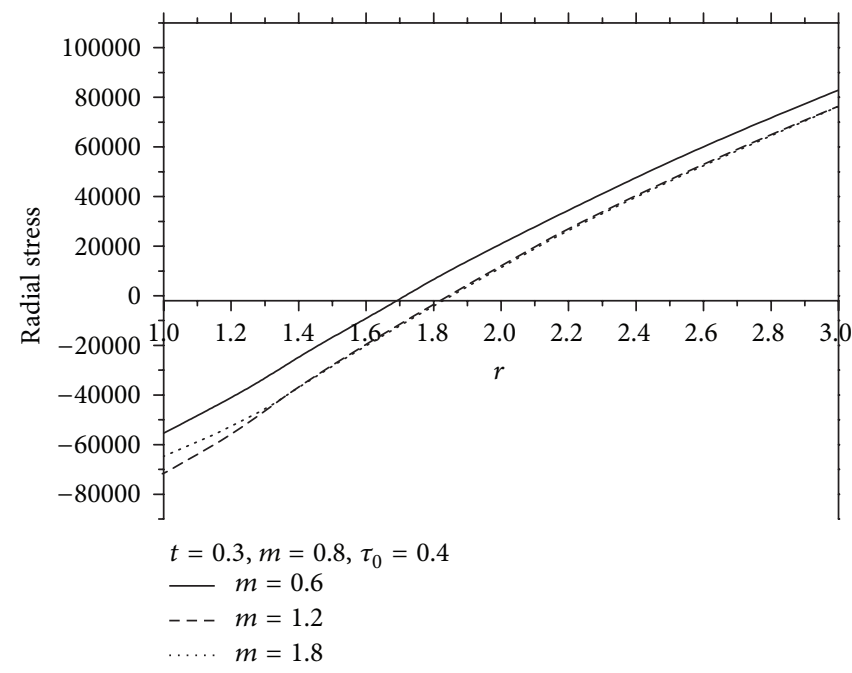

(b)

FIGURE 5: Variation of radial stress $\sigma_{r r}$ with radius $r$ (thermoelastic diffusion nonhomogeneity medium).

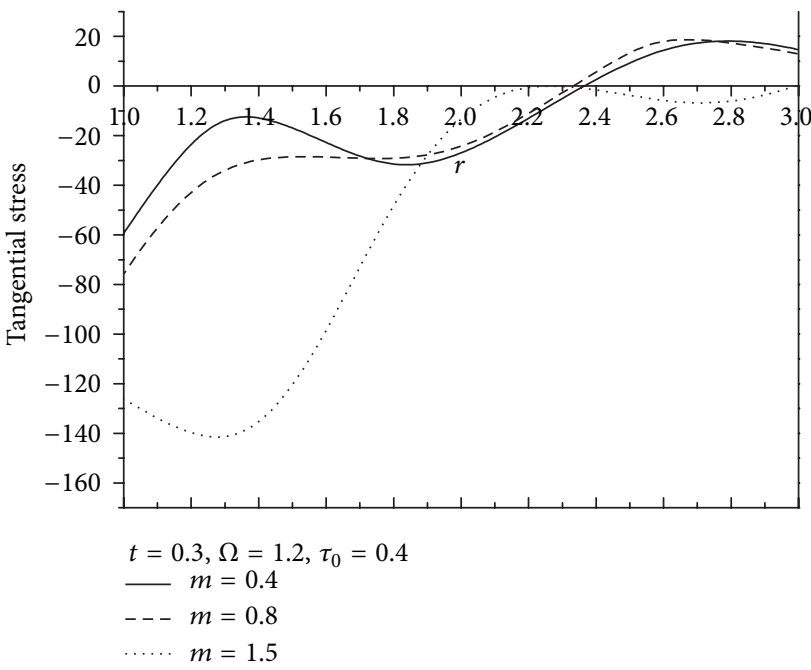

(a)

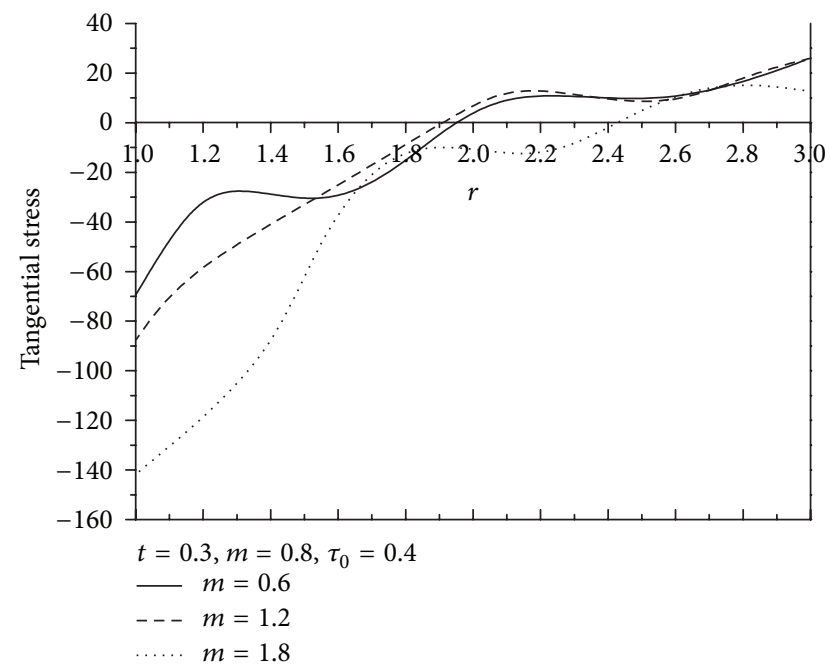

(b)

FiguRE 6: Variation of tangential stress $\sigma_{\phi \phi}$ with radius $r$ (thermoelastic diffusion nonhomogeneity medium).

kind of order $1 / 2$. Compatibility between (26) along with (19) and (20) will give rise to
Substituting (26) into (17a) and (17b), we obtain

$$
\begin{aligned}
& T(r, t)=\frac{1}{\sqrt{r}} \sum_{j=1}^{3} B_{j}(\omega) K_{1 / 2}\left(Q_{j} r\right) e^{i \omega t}, \\
& e(r, t)=\frac{1}{\sqrt{r}} \sum_{j=1}^{3} B_{j}^{\prime}(\omega) K_{1 / 2}\left(Q_{j} r\right) e^{i \omega t}, \\
& C(r, t)=\frac{1}{\sqrt{r}} \sum_{j=1}^{3} B_{j}^{\prime \prime}(\omega) K_{1 / 2}\left(Q_{j} r\right) e^{i \omega t} .
\end{aligned}
$$




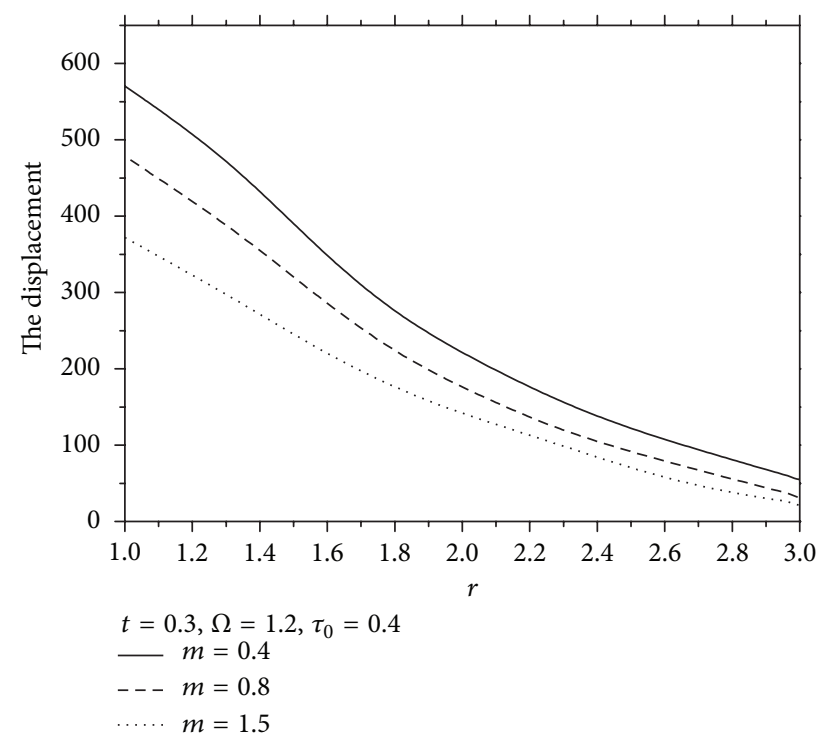

FIGURE 7: Variation of displacement $u$ with radius $r$ (thermoelastic nonhomogeneity medium).

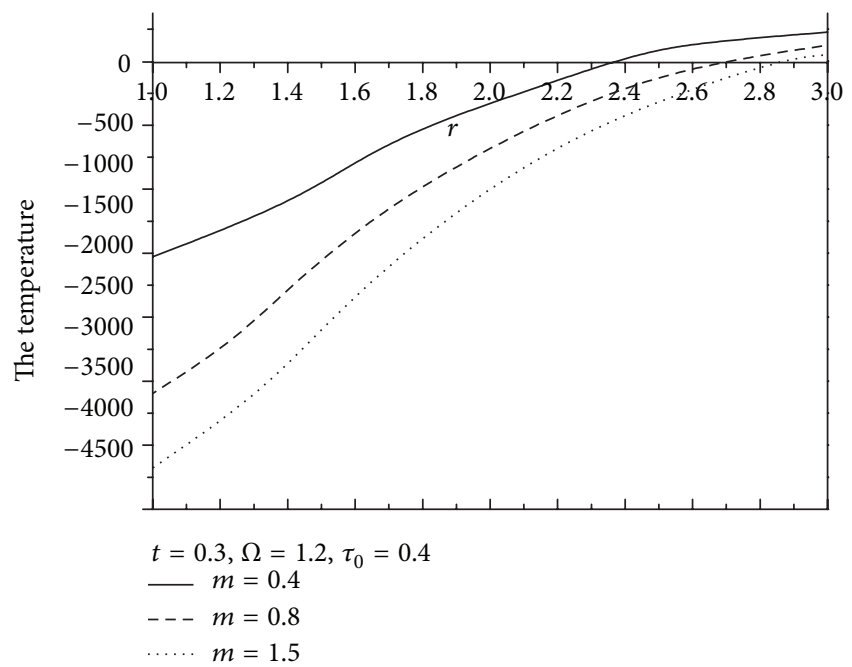

FIGURE 8: Variation of temperature $\theta$ with radius $r$ (thermoelastic nonhomogeneity medium).

Integrating both sides of (29) from $r$ to infinity and assuming that $u(r, t)$ vanishes at infinity, we obtain

$$
\begin{gathered}
u(r, t)=\frac{1}{\sqrt{r}} \sum_{j=1}^{3} \frac{\left(Q_{j}^{2}-k_{1}\right)\left(h_{5} Q_{j}^{2}-h_{6} k_{2}\right)-\varepsilon_{2} k_{1} h_{4} Q_{j}^{2}}{Q_{j}^{2} \varepsilon_{2} k_{1}+k_{1} \varepsilon_{1}\left(h_{5} Q_{j}^{2}-h_{6} k_{2}\right)} \\
\times B_{j}(\omega) K_{3 / 2}\left(Q_{j} r\right) e^{i \omega t} .
\end{gathered}
$$

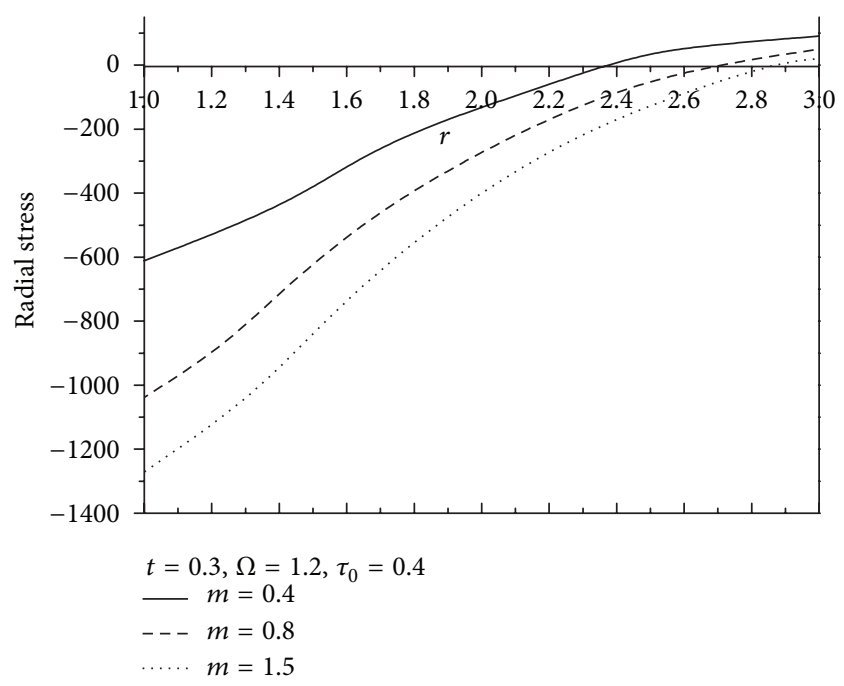

FIGURE 9: Variation of radial stress $\sigma_{r r}$ with radius $r$ (thermoelastic nonhomogeneity medium).

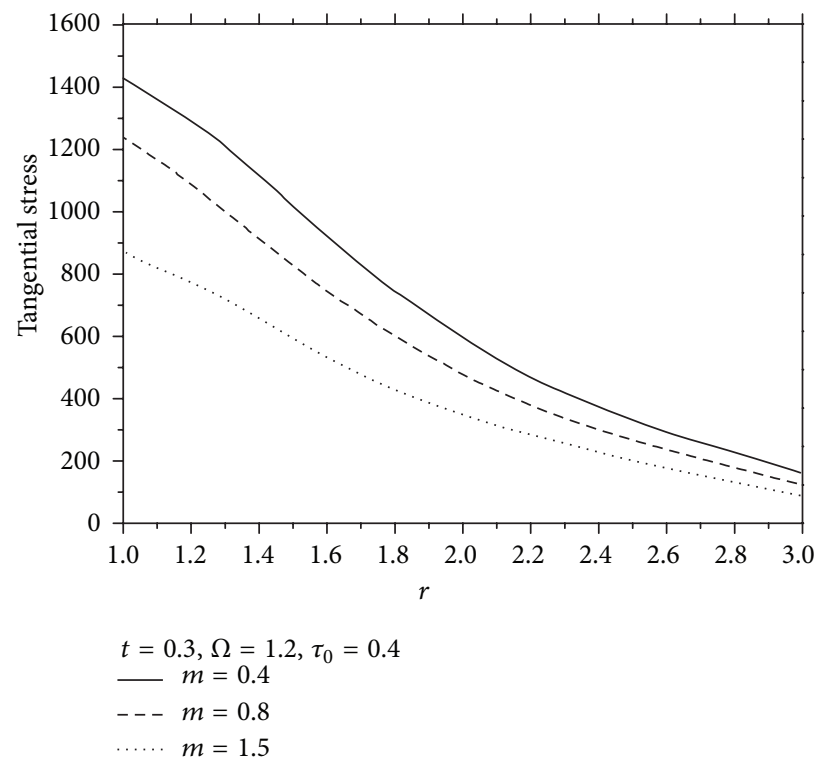

FIGURE 10: Variation of tangential stress $\sigma_{\phi \phi}$ with radius $r$ (thermoelastic nonhomogeneity medium).

From (13a), (13b), and (13c)-(14), we get

$$
\begin{aligned}
& \sigma_{r r}=\frac{1}{\sqrt{r}} \sum_{j=1}^{3} B_{j}(\omega) \frac{\left(Q_{j}^{2}-k_{1}\right)\left(h_{5} Q_{j}^{2}-h_{6} k_{2}\right)-\varepsilon_{2} k_{1} h_{4} Q_{j}^{2}}{Q_{j}^{2} \varepsilon_{2} k_{1}+k_{1} \varepsilon_{1}\left(h_{5} Q_{j}^{2}-h_{6} k_{2}\right)} \\
& \times\left\{\left[\left(h_{1}+h_{2}\right)-\frac{Q_{j}^{2} \varepsilon_{2} k_{1}+k_{1} \varepsilon_{1}\left(h_{5} Q_{j}^{2}-h_{6} k_{2}\right)}{\left(Q_{j}^{2}-k_{1}\right)\left(h_{5} Q_{j}^{2}-h_{6} k_{2}\right)-\varepsilon_{2} k_{1} h_{4} Q_{j}^{2}}\right]\right. \\
&\left.\times K_{1 / 2}\left(Q_{j} r\right)-\frac{h_{1}}{r} K_{3 / 2}\left(Q_{j} r\right)\right\} e^{i \omega t},
\end{aligned}
$$




$$
\begin{aligned}
& \sigma_{\phi \phi}=\sigma_{\theta \theta} \\
& =\frac{1}{\sqrt{r}} \sum_{j=1}^{3} B_{j}(\omega) \\
& \times\left\{\frac{h_{1}}{r} \frac{\left(Q_{j}^{2}-k_{1}\right)\left(h_{5} Q_{j}^{2}-h_{6} k_{2}\right)-\varepsilon_{2} k_{1} h_{4} Q_{j}^{2}}{Q_{j}^{2} \varepsilon_{2} k_{1}+k_{1} \varepsilon_{1}\left(h_{5} Q_{j}^{2}-h_{6} k_{2}\right)}\right. \\
& \times K_{3 / 2}\left(Q_{j} r\right) \\
& +\left[h_{2} \frac{\left(Q_{j}^{2}-k_{1}\right)\left(h_{5} Q_{j}^{2}-h_{6} k_{2}\right)-\varepsilon_{2} k_{1} h_{4} Q_{j}^{2}}{Q_{j}^{2} \varepsilon_{2} k_{1}+k_{1} \varepsilon_{1}\left(h_{5} Q_{j}^{2}-h_{6} k_{2}\right)}\right. \\
& \left.-1-\frac{\varepsilon_{1} k_{1} h_{4} Q_{j}^{2}+Q_{j}^{2}\left(Q_{j}^{2}-k_{1}\right)}{Q_{j}^{2} \varepsilon_{2} k_{1}+k_{1} \varepsilon_{1}\left(h_{5} Q_{j}^{2}-h_{6} k_{2}\right)}\right] \\
& \left.\times K_{1 / 2}\left(Q_{j} r\right)\right\} e^{i \omega t} \\
& P=\frac{1}{\sqrt{r}} \sum_{j=1}^{3} B_{j}(\omega)\left\{-\frac{\left(Q_{j}^{2}-k_{1}\right)\left(h_{5} Q_{j}^{2}-h_{6} k_{2}\right)-\varepsilon_{2} k_{1} h_{4} Q_{j}^{2}}{Q_{j}^{2} \varepsilon_{2} k_{1}+k_{1} \varepsilon_{1}\left(h_{5} Q_{j}^{2}-h_{6} k_{2}\right)}\right. \\
& \left.+h_{3} \frac{\varepsilon_{1} k_{1} h_{4} Q_{j}^{2}+Q_{j}^{2}\left(Q_{j}^{2}-k_{1}\right)}{Q_{j}^{2} \varepsilon_{2} k_{1}+k_{1} \varepsilon_{1}\left(h_{5} Q_{j}^{2}-h_{6} k_{2}\right)}-h_{4}\right\} \\
& \times K_{1 / 2}\left(Q_{j} r\right) e^{i \omega t} \text {. }
\end{aligned}
$$

\section{Particular Case}

If we neglect the initial stress and diffusion effects by eliminating (3) and (8) and putting $P_{1}=\beta_{2}=C=0$ in (4) and (6), we get $(T, e), u(r, t), \sigma_{r r}, \sigma_{\phi \phi}$, and $\sigma_{\theta \theta}$ :

$$
j=1,2,3 \text {. }
$$




$$
\begin{aligned}
\sigma_{\phi \phi}=\sigma_{\theta \theta}=-\{ & \left(\alpha_{1}\left(\frac{r^{2} \lambda_{1}^{2}+2 r \lambda_{1}+2}{r^{3} \lambda_{1}^{3}}\right)-\left(\alpha_{2}-1\right)\right) \\
& \times A(\omega) e^{-\lambda_{1} r} \\
+ & {\left[\alpha_{1}\left(\frac{r^{2} \lambda_{2}^{2}+2 r \lambda_{2}+2}{r^{3} \lambda_{2}^{3}}\right)-\left(\alpha_{2}-1\right)\right] } \\
& \left.\times B(\omega) e^{-\lambda_{2} r}\right\} e^{i \omega t} .
\end{aligned}
$$

Using the boundary conditions, we obtain

$$
\begin{gathered}
A(\omega)=\frac{-h_{2} T_{0}}{h_{1} e^{-\lambda_{2} a}-h_{2} e^{-\lambda_{1} a}}, \quad B(\omega)=\frac{h_{1} T_{0}}{h_{1} e^{-\lambda_{2} a}-h_{2} e^{-\lambda_{1} a}}, \\
C(\omega)=\frac{\left(h_{2} h_{3}-h_{1} h_{4}\right) \theta_{0}}{h_{1} e^{-\lambda_{2} a}-h_{2} e^{-\lambda_{1} a}}, \\
h_{1}=\alpha_{1}\left(\frac{4+4 a \lambda_{1}+2 a^{2} \lambda_{1}^{2}+a^{3} \lambda_{1}^{3}}{a^{3} \lambda_{1}^{3}}\right. \\
h_{2}=\alpha_{1}\left(\frac{4+4 a \lambda_{2}+2 a^{2} \lambda_{2}^{2}+a^{3} \lambda_{2}^{3}}{a^{3} \lambda_{2}^{3}}\right. \\
\alpha_{3}=\left(\frac{\alpha^{2} \lambda_{1}^{2}+2 a \lambda_{1}+2}{a^{2} \lambda_{1}^{3}}\right) e^{-\lambda_{1} a}, \\
h_{4}=\left(\frac{a^{2} \lambda_{2}^{2}+2 a \lambda_{2}+2}{a^{2} \lambda_{2}^{3}}\right) e^{-\lambda_{2} a} . \\
\left.+\left(\alpha_{2}-1-\mu_{e} H_{\phi}^{2} \lambda_{2}\right)\right) e^{-\lambda_{2} a},
\end{gathered}
$$

\section{Numerical Results and Discussion}

For the purposes of numerical evaluations. The copper material was chosen. The constants of the problem given by Aouadi [24], Sokolnikoff [34] and Thomas [35] are

$$
\begin{gathered}
\mu=3.86 \times 10^{10} \mathrm{~kg} / \mathrm{ms}^{3}, \quad \lambda=7.76 \times 10^{10} \mathrm{~kg} / \mathrm{ms}^{3}, \\
\rho=8954 \mathrm{~kg} / \mathrm{m}^{3}, \quad c_{v}=383.1 \mathrm{~J} / \mathrm{kg} \cdot \mathrm{K}, \\
\alpha_{t}=1.78 \times 10^{-5} \mathrm{~K}^{-1}, \quad \alpha_{c}=1.98 \times 10^{-4} \mathrm{~m}^{3} / \mathrm{kg}, \\
k=386 \mathrm{~W} / \mathrm{mK}, \quad D=0.85 \times 10^{8} \mathrm{~kg} \cdot \mathrm{s} / \mathrm{m}^{3}, \\
T_{0}=293 \mathrm{~K}, \quad c=1.2 \times 10^{4} \mathrm{~m}^{2} / \mathrm{s}^{2} \mathrm{~K}, \\
b=0.9 \times 10^{6} \mathrm{~m}^{5} / \mathrm{s}^{2} \mathrm{~kg}, \quad \eta_{0}=8886.73 \mathrm{~s} / \mathrm{m}^{2} .
\end{gathered}
$$

Using the above values, we get $\mu_{e}=1, \theta_{0}=1, P_{0}=1$, $a=2, \omega=9.5, H=0.7 \times 10^{-5}, \tau_{0}=0.1$, and $\tau=0.2$.
The values of radial displacement $u$, temperature distribution $\theta$, concentration $C$, stresses $\sigma_{r r}, \sigma_{\phi \phi}$, and chemical potential distribution $P$ for thermoelastic diffusion and thermoelasticity are studied for force thermal source and chemical potential source. The output is plotted in Figures 1-10. Figure 1 shows that the values of chemical potential distribution $P$ have oscillatory behavior with diffusion in the whole range of radius $r$. The effects of nonhomogeneity $m$, rotation $\Omega$, time $t$ and relaxation time $\tau_{0}$ on chemical potential distribution is shifting from the positive into the negative gradually with the radius $r$. Figure 2 shows that the value of concentration distribution $C$ has oscillatory behavior for diffusion in the whole range of radius $r$ under the effects of nonhomogeneity, rotation, and relaxation time, while it is decreasing with an increase of nonhomogeneity $m$. In these figures, it is clear that the distribution has a nonzero value only in the bounded region of space for $t=0.15$ where the infinite speed of propagation is inherent. The effects of nonhomogeneity, rotation $\Omega$, time $t$, and relaxation time $\tau_{0}$ on concentration distribution is shifting from the positive into the negative gradually. This indicates that the equations are satisfied by the concentration $C$ which predict a finite speed of propagation of matter from first medium to another one. Figure 3 shows that the value of temperature distribution $\theta$ has an oscillatory behavior for thermoelastic diffusion in the whole range of the radius $r$, while the solution is notably different inside the sphere. This is due to the fact that, the thermal waves in the coupled theory travel with an infinite speed of propagation as opposed to finite speed in the generalized case. The effects of nonhomogeneity, rotation $\Omega$, time $t$ and relaxation time $\tau_{0}$ on temperature distribution shift from the positive into the negative gradually. This indicates that the heat propagates as a wave with finite velocity. Figure 4 shows that the value of radial displacement $u$ has oscillatory behavior with diffusion in the whole range of radius $r$. These figures indicate that the medium along $r$ undergoes expansion deformation due to the thermal shock, while the other one shows the compressive deformation. The effect of nonhomogeneity, rotation $\Omega$, and relaxation time $\tau_{0}$ on radial displacement becomes large. Increasing the nonhomogeneity, the radial displacement is shifted upward from negative values to positive values. At a given instant, the radial displacement is finite which is due to the effect of nonhomogeneity, rotation, time, and relaxation time. Figures 5 and 6 show the variations of the radial stress $\sigma_{r r}$ and tangential stress $\sigma_{\phi \phi}$ with respect to the radius $r$, respectively. The values of radial stress and tangential stress are increased and decreased due to the diffusion in a nonuniform behavior for all values of the radius $r$. For the values of $\sigma_{r r}$ and $\sigma_{\phi \phi}$, depicting the effect of nonhomogeneity, diffusion, rotation, and relaxation time, it is shown that the radial stress is compressive in its nature.

Figure 7 shows the values of radial displacement $u$ in thermoelastic medium without diffusion. This figure indicates clearly that the radial displacement at the cavity surface tends to zero which agrees with the boundary conditions prescribed. This coincides with the mechanical boundary condition of the cavity, in case of fixed surface. Figure 8 shows the values of temperature distribution $\theta$ without diffusion in the whole range of radius $r$. It was found that the values 
of $\theta$ under effect of nonhomogeneity and rotation $\Omega$ are increase with an increase of nonhomogeneity and rotation $\Omega$ but are decreasing with the increase of the values of $m$. Figures 9 and 10 show the values of radial stress $\sigma_{r r}$ and the tangential stress $\sigma_{\phi \phi}$ without diffusion in the whole range of radius $r$, respectively. It was found that the values of $\sigma_{r r}$ under the effects of nonhomogeneity $m$ and rotation $\Omega$ are increasing with an increase of the values of nonhomogeneity $m$ and rotation $\Omega$, while the values of $\sigma_{r r}$ are decreasing with an increase of nonhomogeneity $m$, while the tangential stress $\sigma_{\phi \phi}$ is decreasing with the increase of the values of nonhomogeneity $m$ and rotation $\Omega$, but the values of $\sigma_{\phi \phi}$ are increasing with an increase of $m$. Due to the complicated nature of the governing equations of the generalized magneto-thermoelastic diffusion theory, the done works in this field are unfortunately limited. The method used in this study provides quite a success in dealing with such problems. This method gives exact solutions in the elastic medium without any restrictions on the actual physical quantities that appear in the governing equations of the considered problem.

\section{Conclusions}

The results presented in this paper will be very helpful for researchers concerned with material science, designers of new materials, and low-temperature physicists, as well as for those working on the development of a theory of hyperbolic propagation of hyperbolic thermodiffusion. Study of the phenomenon of nonhomogeneity, rotation, magnetic field, and diffusion is also used to improve the conditions of oil extractions. It was found that, for values of rotation and nonhomogeneity, the coupled theory and the generalization give close results. The case is quite different when we consider small value of rotation and nonhomogeneity. Comparing Figures 1-6 in case of thermoelastic diffusion medium with the Figures 7-10 in case of thermoelastic medium, it was found that $u, \sigma_{r r}, \sigma_{\phi \phi}, C$, and $P$ have the same behavior in both media. But with the passage of nonhomogeneity and rotation, the numerical values of $u, \sigma_{r r}, \sigma_{\phi \phi}, C$, and $P$ in thermoelastic diffusion medium are large in comparison with those in thermoelastic medium due to the influences of nonhomogeneity, magnetic field, rotation, and mass diffusion.

\section{Acknowledgment}

This article was funded by the Deanship of Scientific Research (DSR), King Abdulaziz University, Jeddah. The author, therefore, acknowledge with thanks DSR technical and financial support.

\section{References}

[1] D. Motreanu and M. Sofonea, "Evolutionary variational inequalities arising in quasistatic frictional contact problems for elastic materials," Abstract and Applied Analysis, vol. 4, no. 4, pp. 255-279, 1999.

[2] T. M. Atanacković, S. Konjik, L. Oparnica, and D. Zorica, "Thermodynamical restrictions and wave propagation for a class of fractional order viscoelastic rods," Abstract and Applied Analysis, vol. 2011, Article ID 975694, 32 pages, 2011.

[3] R. Fares, G. P. Panasenko, and R. Stavre, "A viscous fluid flow through a thin channel with mixed rigid-elastic boundary: variational and asymptotic analysis," Abstract and Applied Analysis, vol. 2012, Article ID 152743, 47 pages, 2012.

[4] M. Marin, R. P. Agarwal, and S. R. Mahmoud, "Modeling a microstretch thermoelastic body with two temperatures," Abstract and Applied Analysis, vol. 2013, Article ID 583464, 7 pages, 2013.

[5] M. N. M. Allam, A. M. Zenkour, and E. R. Elazab, "The rotating inhomogeneous elastic cylinders of variable-thickness and density," Applied Mathematics \& Information Sciences, vol. 2, no. 3, pp. 237-257, 2008.

[6] A. M. Abd-Alla, S. R. Mahmoud, and N. A. Al-Shehri, "Effect of the rotation on a non-homogeneous infinite cylinder of orthotropic material," Applied Mathematics and Computation, vol. 217, no. 22, pp. 8914-8922, 2011.

[7] A. M. Abd-Alla, S. R. Mahmoud, and S. M. Abo-Dahab, "On problem of transient coupled thermoelasticity of an annular fin," Meccanica, vol. 47, no. 5, pp. 1295-1306, 2012.

[8] A. M. Abd-Alla, S. R. Mahmoud, and A. M. El-Naggar, "Analytical solution of electro-mechanical wave propagation in long bones," Applied Mathematics and Computation, vol. 119, no. 1, pp. 77-98, 2001.

[9] H. W. Lord and Y. Shulman, "A generalized dynamical theory of thermo-elasticity," Journal of the Mechanics and Physics of Solids, vol. 7, pp. 71-75, 1967.

[10] R. S. Dhaliwal and H. H. Sherief, "Generalized thermoelasticity for anisotropic media," Quarterly of Applied Mathematics, vol. 33, no. 1, pp. 1-8, 1980.

[11] A. M. Abd-Alla and S. R. Mahmoud, "Magneto-thermoelastic problem in rotating non-homogeneous orthotropic hollow cylinder under the hyperbolic heat conduction model," Meccanica, vol. 45, no. 4, pp. 451-462, 2010.

[12] S. R. Mahmoud, "Wave propagation in cylindrical poroelastic dry bones," Applied Mathematics \& Information Sciences, vol. 4, no. 2, pp. 209-226, 2010.

[13] R. Kumar and S. Devi, "Deformation in porous thermoelastic material with temperature dependent properties," Applied Mathematics \& Information Sciences, vol. 5, no. 1, pp. 132-147, 2011.

[14] M. I. A. Othman, "Lord-Shulman theory under the dependence of the modulus of elasticity on the reference temperature in twodimensional generalized thermoelasticity," Journal of Thermal Stresses, vol. 25, no. 11, pp. 1027-1045, 2009.

[15] R. Kumar and R. R. Gupta, "Deformation due to inclined load in an orthotropic micropolar thermoelastic medium with two relaxation times," Applied Mathematics \& Information Sciences, vol. 4, no. 3, pp. 413-428, 2010.

[16] A. E. Green and K. A. Lindsay, "Thermoelasticity," Journal of Elasticity, vol. 2, no. 1, pp. 1-7, 1972.

[17] A. M. Abd-Alla, G. A. Yahya, and S. R. Mahmoud, "Radial vibrations in a non-homogeneous orthotropic elastic hollow sphere subjected to rotation," Journal of Computational and Theoretical Nanoscience, vol. 10, no. 2, pp. 455-463, 2013.

[18] A. M. Abd-Alla, S. M. Abo-Dahab, S. R. Mahmoud, and T. A. AlThamalia, "Influence of the rotation and gravity field on stonely waves in a non-homogeneous orthotropic elastic medium," Journal of Computational and Theoretical Nanoscience, vol. 10, no. 2, pp. 297-305, 2013. 
[19] H. H. Sherief, F. A. Hamza, and H. A. Saleh, "The theory of generalized thermoelastic diffusion," International Journal of Engineering Science, vol. 42, no. 5-6, pp. 591-608, 2004.

[20] H. H. Sherief and H. A. Saleh, "A half-space problem in the theory of generalized thermoelastic diffusion," International Journal of Solids and Structures, vol. 42, no. 15, pp. 4484-4493, 2005.

[21] B. Singh, "Reflection of SV waves from the free surface of an elastic solid in generalized thermoelastic diffusion," Journal of Sound and Vibration, vol. 291, no. 3-5, pp. 764-778, 2006.

[22] R. Kumar and T. Kansal, "Propagation of Lamb waves in transversely isotropic thermoelastic diffusive plate," International Journal of Solids and Structures, vol. 45, no. 22-23, pp. 58905913, 2008.

[23] P. Ram, N. Sharma, and R. Kumar, "Thermomechanical response of generalized thermoelastic diffusion with one relaxation time due to time harmonic sources," International Journal of Thermal Sciences, vol. 47, no. 3, pp. 315-323, 2008.

[24] M. Aouadi, "A problem for an infinite elastic body with a spherical cavity in the theory of generalized thermoelastic diffusion," International Journal of Solids and Structures, vol. 44, no. 17, pp. 5711-5722, 2007.

[25] A. M. Abd-Alla and S. R. Mahmoud, "Analytical solution of wave propagation in a non-homogeneous orthotropic rotating elastic media," Journal of Mechanical Science and Technology, vol. 26, no. 3, pp. 917-926, 2012.

[26] M. I. A. Othman, S. Y. Atwa, and R. M. Farouk, "The effect of diffusion on two-dimensional problem of generalized thermoelasticity with Green-Naghdi theory," International Communications in Heat and Mass Transfer, vol. 36, no. 8, pp. 857-864, 2009.

[27] R.-H. Xia, X.-G. Tian, and Y.-P. Shen, "The influence of diffusion on generalized thermoelastic problems of infinite body with a cylindrical cavity," International Journal of Engineering Science, vol. 47, no. 5-6, pp. 669-679, 2009.

[28] S. Deswal and K. Kalkal, "A two-dimensional generalized electro-magneto-thermoviscoelastic problem for a half-space with diffusion," International Journal of Thermal Sciences, vol. 50, no. 5, pp. 749-759, 2011.

[29] A. M. Abd-Alla and S. M. Abo-Dahab, "Time-harmonic sources in a generalized magneto-thermo-viscoelastic continuum with and without energy dissipation," Applied Mathematical Modelling, vol. 33, no. 5, pp. 2388-2402, 2009.

[30] S. R. Mahmoud, "Influence of rotation and generalized magneto-thermoelastic on rayleigh waves in a granular medium under effect of initial stress and gravity field," Meccanica, vol. 47, no. 7, pp. 1561-1579, 2012.

[31] A. M. Abd-Alla, S. M. Abo-Dahab, H. A. Hammad, and S. R. Mahmoud, "On generalized magneto-thermoelastic rayleigh waves in a granular medium under the influence of a gravity field and initial stress," Journal of Vibration and Control, vol. 17, no. 1, pp. 115-128, 2011.

[32] A. M. Abd-Alla and S. R. Mahmoud, "On problem of radial vibrations in non-homogeneity isotropic cylinder under influence of initial stress and magnetic field," Journal of Vibration and Control, vol. 19, no. 9, pp. 1283-1293, 2013.

[33] J. D. Kraus, Electromagnetics, Mc Graw-Hill, New York, NY, USA, 1984.

[34] I. S. Sokolnikoff, Mathematical Theory of Elasticity, Dover, New York, NY, USA, 1946.

[35] L. Thomas, Fundamentals of Heat Transfer, Prentice-Hall, Englewood Cliffs, NJ, USA, 1980. 


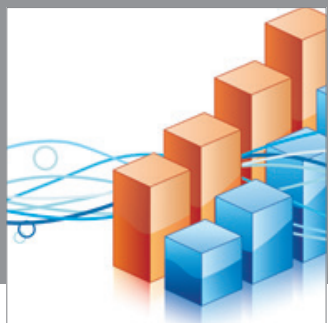

Advances in

Operations Research

mansans

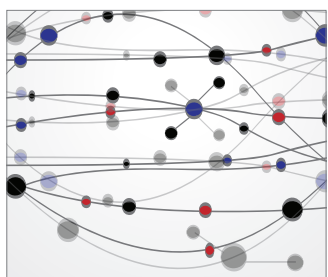

The Scientific World Journal
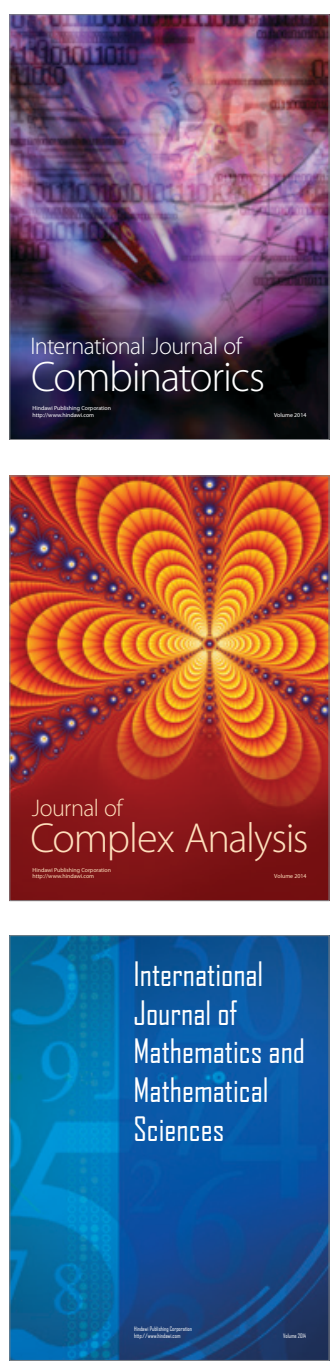
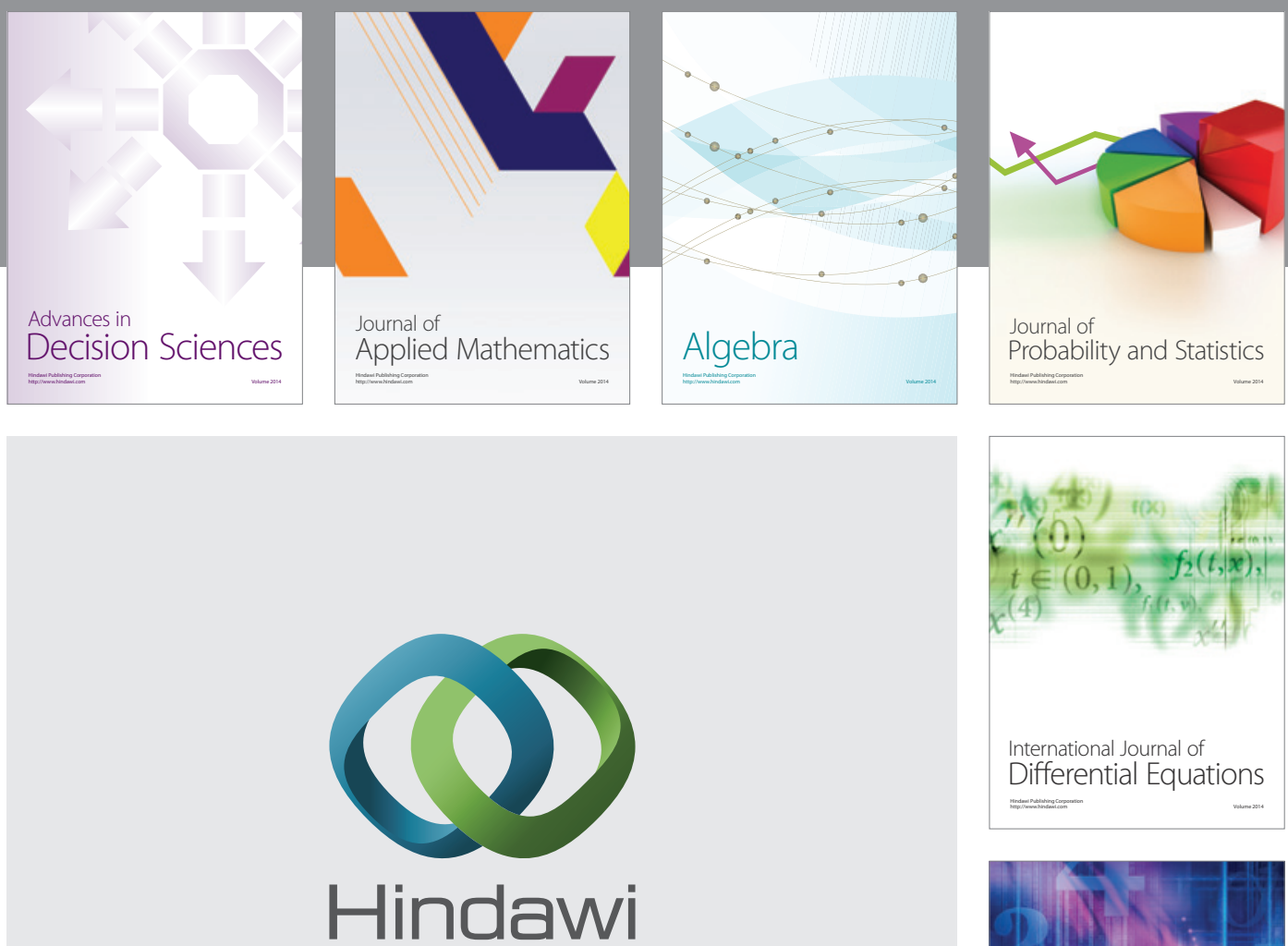

Submit your manuscripts at http://www.hindawi.com
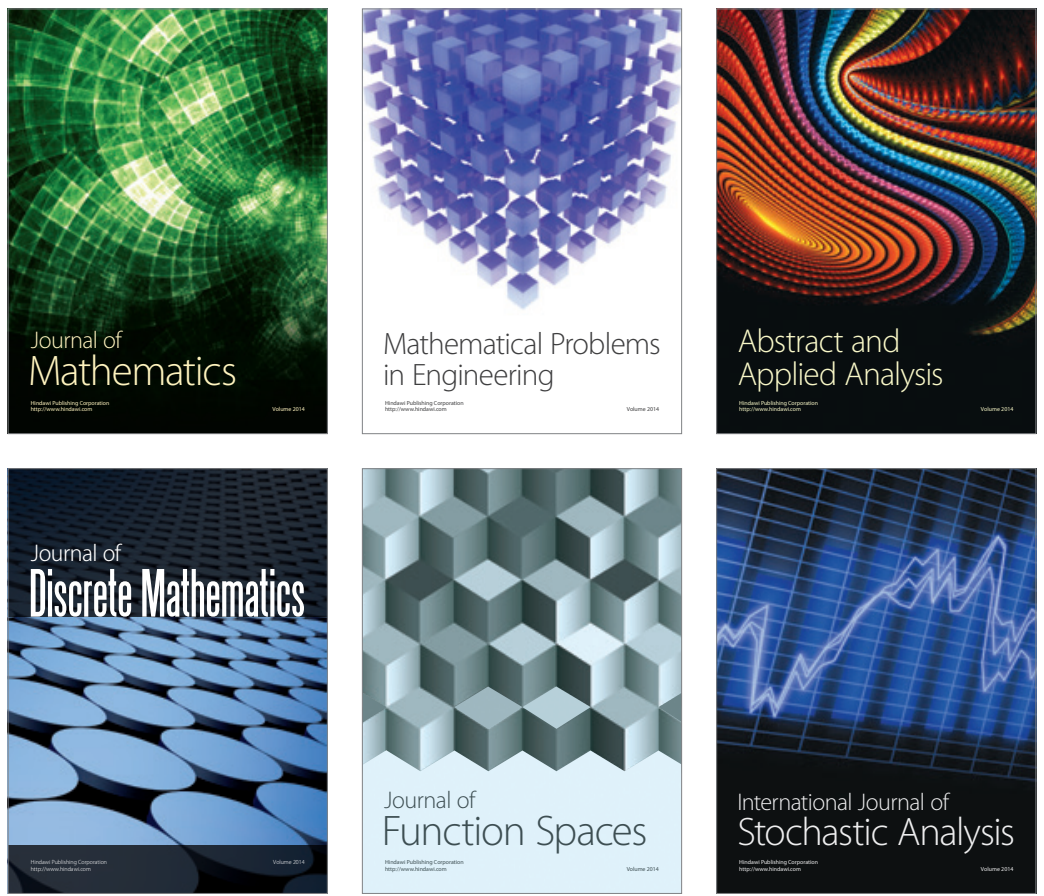

Journal of

Function Spaces

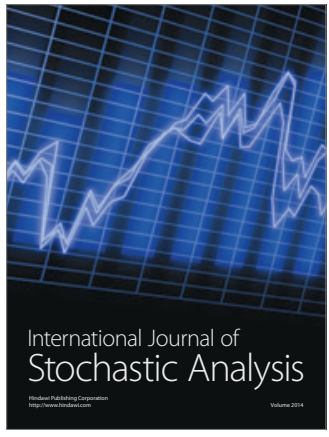

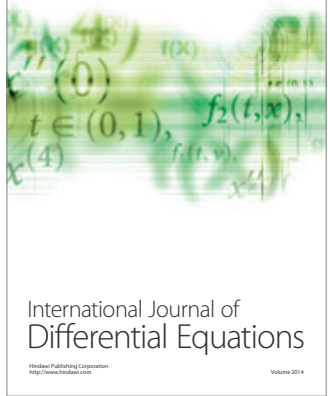
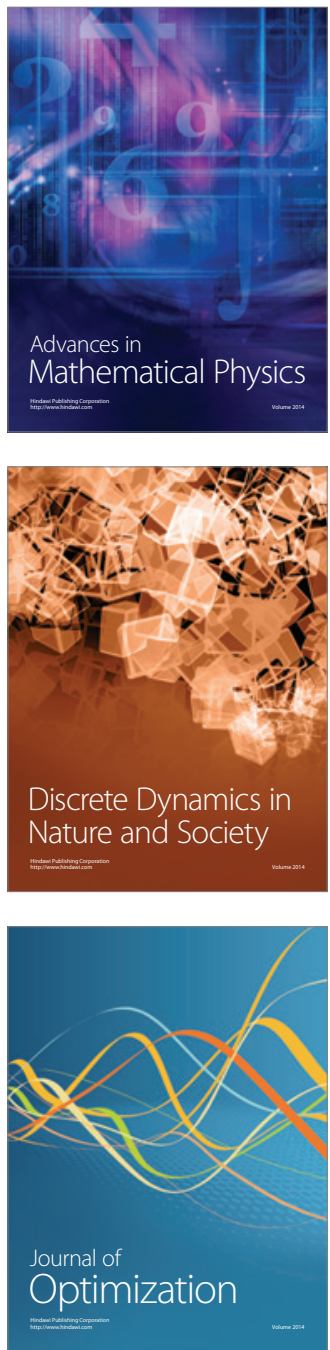